\title{
The development, implementation and evaluation of interventions to reduce workplace sitting: a qualitative systematic review and evidence-based operational framework
}

\author{
Kelly Mackenzie $^{1 *}$ (B), Elizabeth Such ${ }^{1}$, Paul Norman $^{2}$ and Elizabeth Goyder ${ }^{1}$
}

\begin{abstract}
Background: Prolonged sitting is associated with increased risks of cardiovascular disease, Type 2 diabetes, some cancers, musculoskeletal disorders and premature mortality. Workplaces contribute to a large proportion of daily sitting time, particularly among office-based workers. Interventions to reduce workplace sitting therefore represent important public health initiatives. Previous systematic reviews suggest such interventions can be effective but have reported wide variations. Further, there is uncertainty as to whether effectiveness in controlled trials can be replicated when implemented outside the research setting. The aims of this review are to identify factors important for the implementation of workplace sitting interventions and to translate these findings into a useful operational framework to support the future implementation of such interventions.
\end{abstract}

Methods: A qualitative systematic review was conducted. Four health and social science databases were searched for studies set in the workplace, with office-based employees and with the primary aim of reducing workplace sitting. Extracted data were primarily from author descriptions of interventions and their implementation. Inductive thematic analysis and synthesis was undertaken.

Results: Forty studies met the inclusion criteria. Nine descriptive themes were identified from which emerged three higher-order analytical themes, which related to the development, implementation and evaluation of workplace sitting interventions. Key findings included: the importance of grounding interventions in theory; utilising participative approaches during intervention development and implementation; and conducting comprehensive process and outcome evaluations. There was a general under-reporting of information relating to the context within which workplace sitting interventions were implemented, such as details of local organisation processes and structures, as well as the wider political and economic landscape, which if present would aid the translation of knowledge into "real-world" settings.

Conclusions: These findings provided the basis for an operational framework, which is a representation of all nine descriptive themes and three higher-order analytical themes, to support workplace sitting intervention development, implementation and evaluation. Once tested and refined, this framework has the potential to be incorporated into a practical toolkit, which could be used by a range of organisations to develop, implement and evaluate their own interventions to reduce workplace sitting time amongst staff.

Keywords: Sedentary behaviour, Sitting time, Workplace, Occupation, Intervention development, Implementation, Evaluation, Framework, Qualitative systematic review

\footnotetext{
* Correspondence: kelly.mackenzie@sheffield.ac.uk

'School of Health and Related Research, University of Sheffield, Regent Street,

Sheffield S1 4DA, UK

Full list of author information is available at the end of the article
}

(c) The Author(s). 2018 Open Access This article is distributed under the terms of the Creative Commons Attribution 4.0 International License (http://creativecommons.org/licenses/by/4.0/), which permits unrestricted use, distribution, and reproduction in any medium, provided you give appropriate credit to the original author(s) and the source, provide a link to the Creative Commons license, and indicate if changes were made. The Creative Commons Public Domain Dedication waiver (http://creativecommons.org/publicdomain/zero/1.0/) applies to the data made available in this article, unless otherwise stated. 


\section{Background}

Sedentary behaviour, defined as any waking behaviour with an energy expenditure of $\leq 1.5$ Metabolic Equivalents while in a sitting, reclining or lying posture [1], when carried out for prolonged periods of time has been identified as an important public health concern [2]. Prolonged sedentary behaviour has been shown to be associated with an increased risk of a range of health issues, including: cardiovascular disease [3-5]; metabolic syndrome/type 2 diabetes [3, 5-8]; obesity [9]; hypertension [10]; some cancers [11]; depression [12]; musculoskeletal problems [13]; and premature mortality [5, 8, 14]. Sedentary behaviour is most commonly assessed as time spent sitting.

Over recent decades, particularly in the Global North, the workplace has been increasingly associated with prolonged sitting behaviours due to advances in technology and computer-based tasks [15]. Observational studies suggest that office-based workers in England spend more than $60 \%$ of their total daily sitting time at work [16]. In response, a number of interventions to reduce workplace sitting have been developed and tested. Systematic reviews investigating the effectiveness of workplace sitting interventions have produced mixed results [17-22]. These reviews suggest that, at least in the short-term, intervention strategies that use sit-stand/standing desks, either as alone or as part of a multi-component intervention, may be effective, although the reductions in workplace sitting time are variable [17-22]. Ergonomic interventions such as sit-stand desks can be associated with high upfront costs. This may represent a significant barrier to uptake for many employers, despite some evidence to suggest these initial costs may be offset by reduced sickness and increased productivity in the future $[23,24]$. Other intervention strategies such as educational or behavioural strategies or changes to organisational policies may not require the same level of financial investment, but have demonstrated inconsistent results $[17,18,20]$.

The Medical Research Council (MRC) has highlighted the importance of understanding factors which may affect implementation of complex interventions [25]. Interventions may be ineffective due to poor design, but they may also be ineffective due to poor implementation. Thus, an intervention that has been developed and shown to be effective in one context, may not necessarily be effective when transferred to another. This may be particularly true for workplaces given the variations that exist in terms of size, sector, organisational structure and culture. In order to fully understand what works, it is essential to gain an understanding of factors that may influence implementation of "sit less" interventions in different workplaces. This information, in turn, would support the implementation of such interventions, allowing policy-makers and practitioners to determine whether an intervention will fit their specific context. To date, there are no systematic reviews exploring factors influencing the implementation of office-based sitting interventions. Therefore, this paper aims to: firstly, systematically review the literature to identify factors important for the implementation of interventions to reduce sitting time amongst office-based workers; and secondly, use these findings to develop an operational framework to support the future implementation of "sit less" interventions.

\section{Methods}

A qualitative systematic review was undertaken. The review protocol was published via PROSPERO (ID: CRD42016052703), although there were some iterative alterations to the methods outlined in the protocol which are detailed in Additional File 1. The preferred reporting items for systematic reviews and meta-analyses (PRISMA) guidelines were used [26].

\section{Search strategy}

Four electronic databases covering a wide range of relevant sources were searched in January 2017: Web of Science Core Collection; MEDLINE; PsycINFO; CINAHL. In addition, citation searches were carried out by examining the reference lists of relevant reviews to identify further studies. Grey literature was also searched for by reviewing Google, Google Scholar and Mendeley. The search strategy focussed on terms relating to employees and workplaces (population and setting), "sit less" interventions, and outcomes that reduced sedentary behaviour. The complete strategy is shown in Additional File 2.

Study selection was initially conducted by the lead author. This process involved five stages as outlined in Fig. 1. To ensure accuracy and consistency of study selection, a $20 \%$ random sample of the papers was independently checked by two additional reviewers at the title review stage. Disagreements were resolved by discussion between the reviewers until consensus was reached. Thereafter, the lead author determined study eligibility, but for those papers where the decision to include was unclear at the full-text review stage, a second reviewer independently checked papers and again, any disagreements were discussed until consensus was reached.

\section{Inclusion criteria}

A broad range of study designs were required to provide rich data in terms of implementation factors from both experimental and observational studies. Study designs therefore included: randomised-controlled trials (RCTs), uncontrolled or controlled trials, before and 


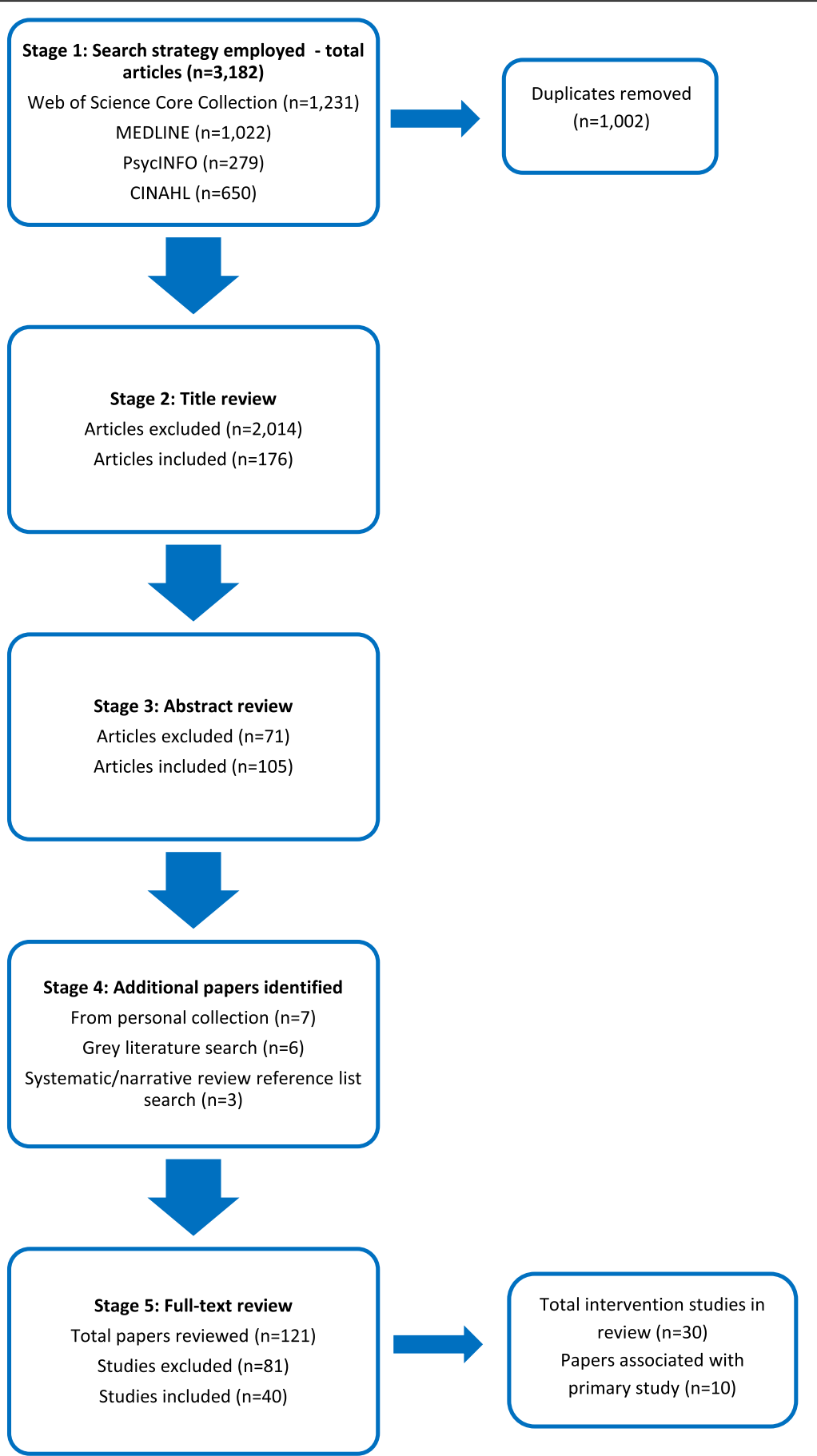

Fig. 1 PRISMA flowchart of study selection process

after studies, and mixed-methods studies. Participants were employed adults in office-based jobs whose occupations involved spending the majority of their working time sitting, e.g., administration, customer service, help-desk professions, call-centre workers, receptionists, academics. Any intervention with the primary aim to reduce workplace sitting time and with a primary outcome of change in workplace sitting time was included. The primary outcome could be measured either objectively, e.g., accelerometer devices, or subjectively, e.g., self-report. In addition, papers associated with the primary study ("associated papers"), e.g., study protocols, intervention development papers and qualitative papers, were also included in the review. 


\section{Data extraction and synthesis}

A pre-piloted data extraction tool was developed to ensure consistent and rigorous data collection. For each included study, the lead author extracted descriptive data pertaining to: study design, setting (country and organisation type); participant information (sample size and demographic information); intervention description (duration, cost and the intervention development process); and measurement tools used. To further ensure accuracy and consistency of data extraction, a 10\% random sample was coded by an additional reviewer. Data relating to intervention implementation came from either first-order constructs, i.e., direct participant quotes or findings reported by authors that were adequately supported by data from focus group discussions, interviews and surveys (where open question responses were recorded); or second-order constructs, i.e., author interpretations, statements, assumptions and ideas.

A formal assessment of quality of the included studies was conducted in order to potentially explain differences in results of otherwise similar studies (see Additional File 3). However, in line with the increasing understanding that qualitative data should be included in systematic reviews [27, 28], all studies were included in the review regardless of quality assessment. This was to allow the inclusion of qualitative data from a range of study designs that may provide a richer understanding of the factors important for the implementation of interventions to reduce workplace sitting.

A narrative synthesis of the descriptive data relating to study design, setting, participant information, intervention and outcome data was undertaken followed by an inductive thematic synthesis of the qualitative data to identify themes relating to intervention implementation. The synthesis followed the three stages as outlined by Thomas and Harden [27]: coding the text line-by-line, developing descriptive themes, and generating analytical themes. Codes were created inductively to capture the meaning and content of each extracted statement. The coding of the text allowed the translation of concepts from one study to another, but new codes continued to be added with each study that was analysed. Similarities and differences between the codes were then reviewed to allow grouping of the codes into a hierarchical structure where initial descriptive themes were identified. Thereafter, higher-order, analytical themes were generated.

\section{Results}

Forty papers were included in the review, comprising 30 primary intervention studies [23, 29-57] and 10 papers that were associated with the primary intervention studies. These associated papers included two study protocols $[58,59]$, two intervention development papers [60, 61], three qualitative papers [62-64], one additional quantitative paper [65], one descriptive paper [66], and one paper published also as a dissertation report [67]. See Table 1 for an outline of the key characteristics of the primary studies and details of the associated papers and Additional File 4 for the extracted raw data.

\section{Study design and setting}

The 30 primary intervention studies comprised 17 RCTs [30-32, 34-38, 40, 41, 48, 49, 52-55, 57], six non-randomised trials $[23,29,33,39,51,56]$, six pre-post intervention studies [42, 43, 45-47, 50], and one mixed-methods study [44].

Thirteen of the intervention studies were set in Australia [23, 29, 31, 32, 35, 37, 39, 40, 42, 44, 45, 48, 51], six in the United States or Canada [33, 36, 41, 43, 54, 57], five in the United Kingdom [30, 46, 47, 50, 55], four in mainland Europe [34, 38, 52, 53], and two did not explicitly state the country setting $[49,56]$. Fifteen studies were conducted either solely $[29,30,32,34,36$, $41,43,46,47,50,55-57]$, or in part $[35,53]$, within an academic institution. The remaining studies were conducted within a range of other public, private and voluntary sector organisations.

\section{Sample size and participant characteristics}

The combined population of the 30 intervention studies included in this review was 2271 participants, with the total sample size per study ranging from 11 to 317 . All except three of the intervention studies $[37,39,51]$ were female-dominated, ranging from 53 to $100 \%$ of samples. Nineteen of the studies [23, 29, 30, 32, 33, 35, 37, 40, 41, $43-46,50,52,53,55-57]$ had majority participants with tertiary-level education or who were in professional (not administrative) job roles.

\section{Interventions}

Intervention duration ranged from one day to 12 months. Three studies did not clearly document intervention duration $[41,52,53]$. Two natural experiments $[44,45]$ involved the evaluation of permanent interventions.

The majority of studies evaluated interventions which involved the use of ergonomic interventions such as sit-stand desks or height-adjustable treadmill desks, either alone $[29,30,40,44,56]$ or in combination with other intervention components such as the provision of educational information $[35,42,47,51,52]$, prompts $[38,51,52,54]$, promotion of other "sit less" initiatives, e.g. walk and talk meetings [48], the use of health coaches/workplace champions [23, 31, 32, 52], individualised feedback [23, 31, 32], or as part of a wider health and wellbeing programme [33]. Other interventions included: automated web-based programmes with a range of support strategies [34, 53]; use of a wrist-worn device to disrupt sedentary behaviour [41]; environmental 


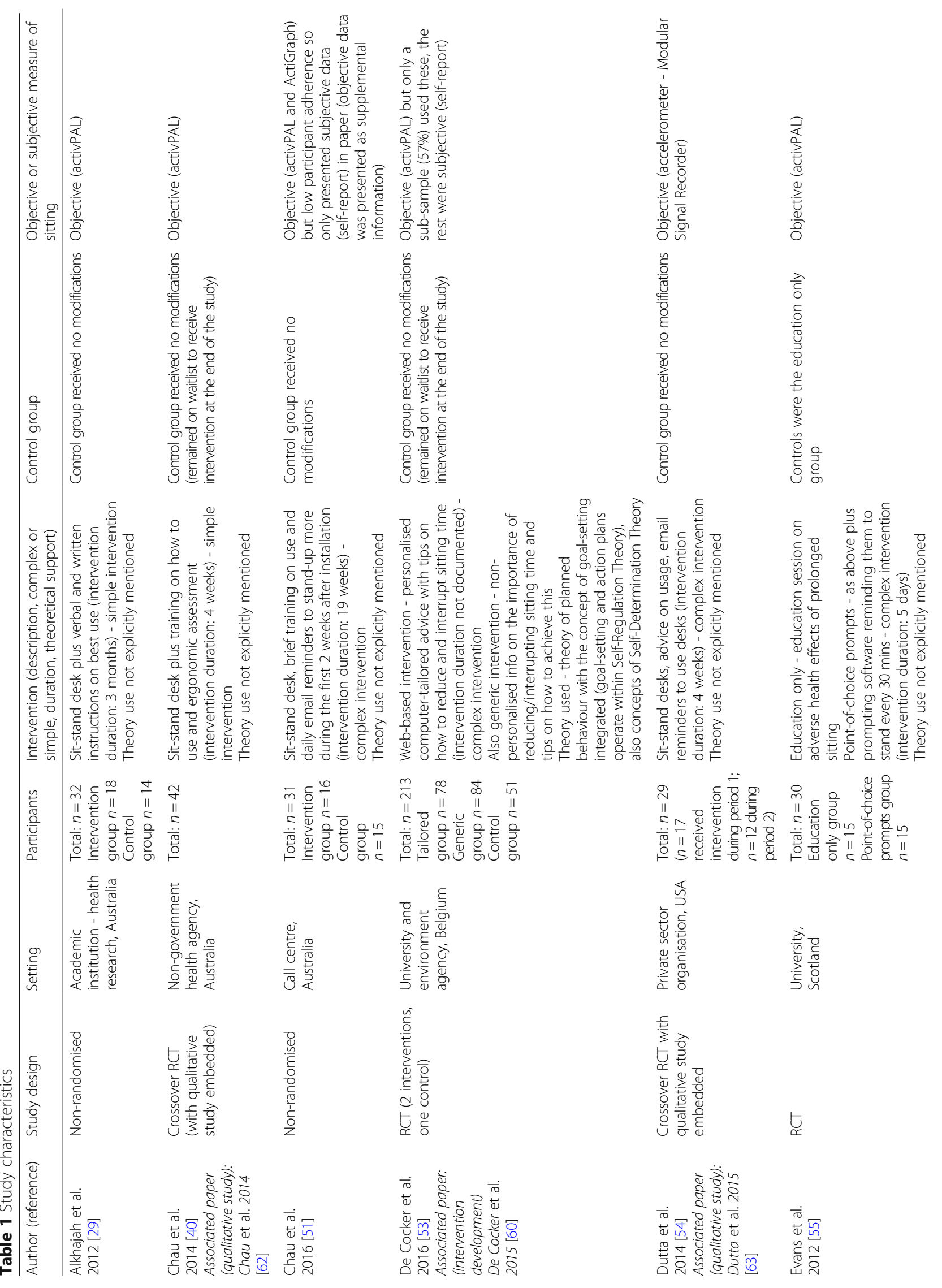




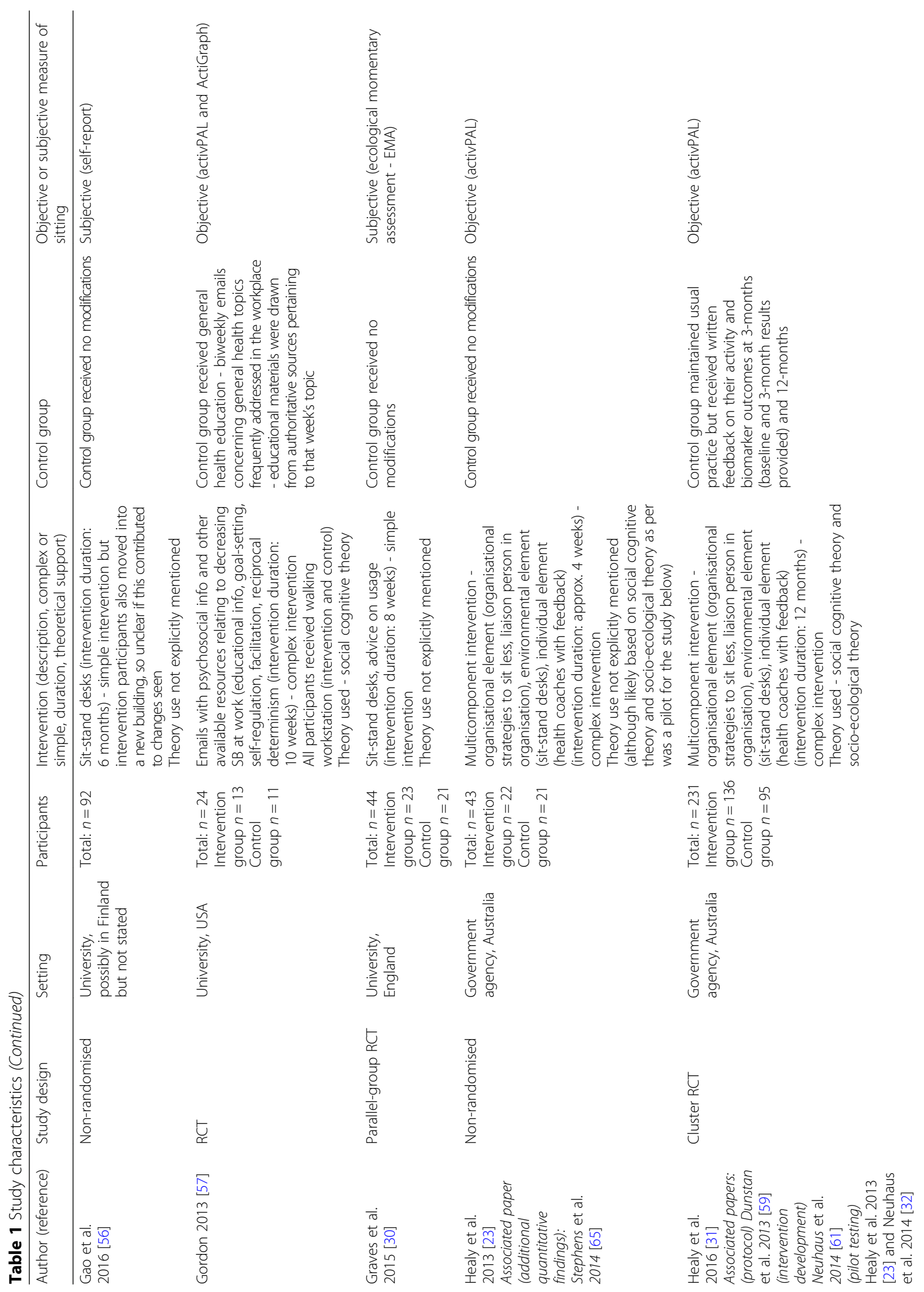




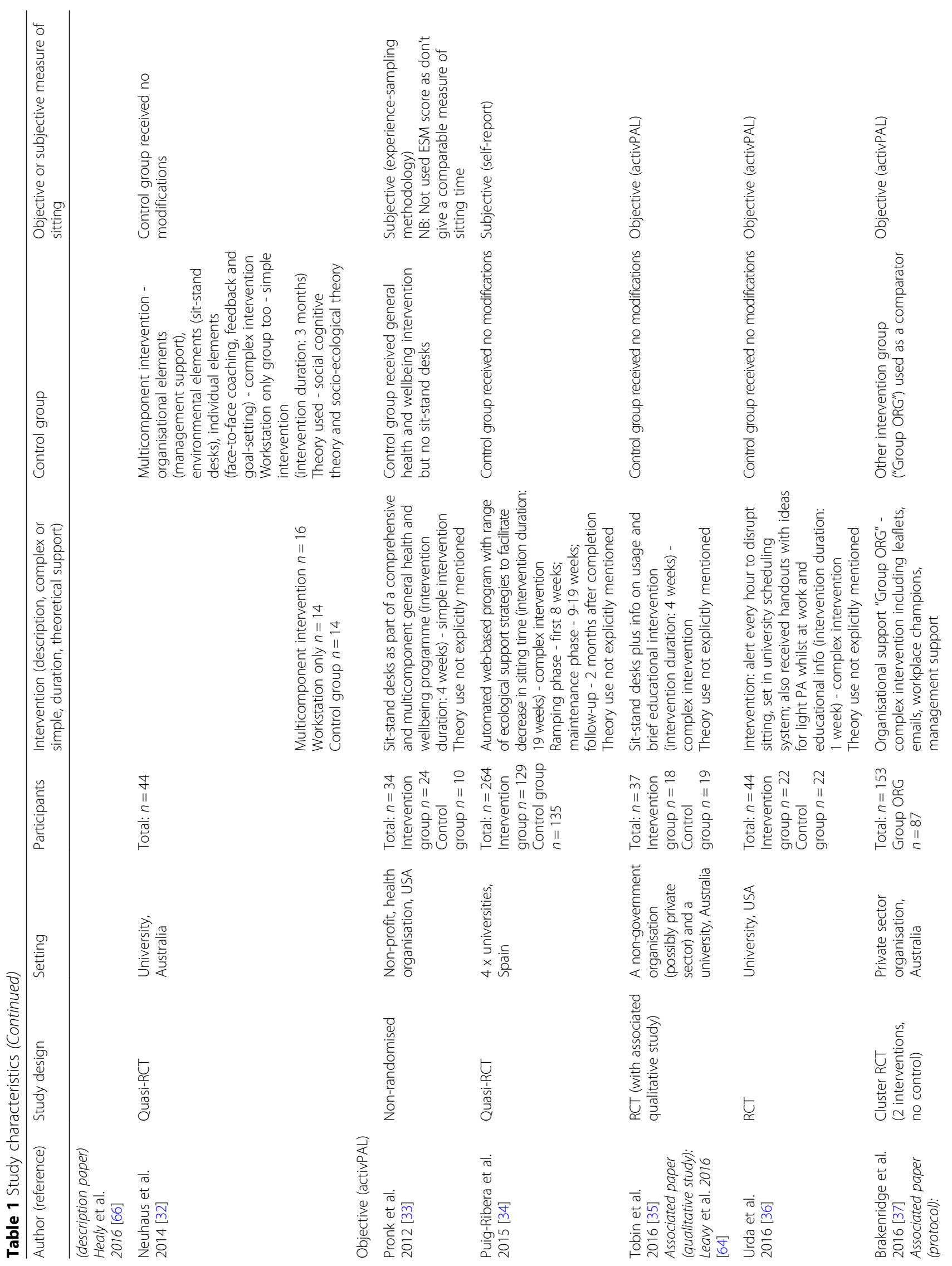




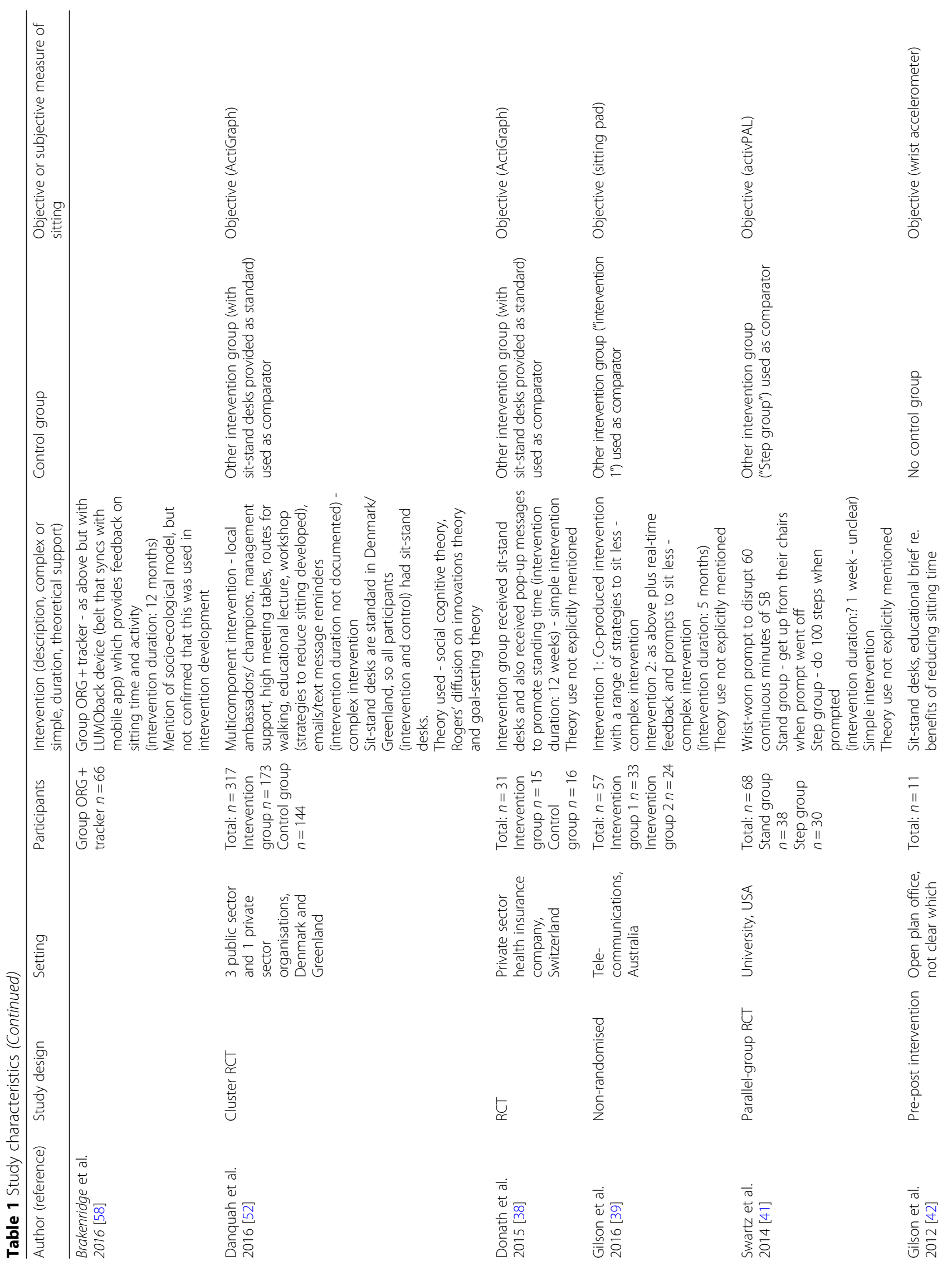




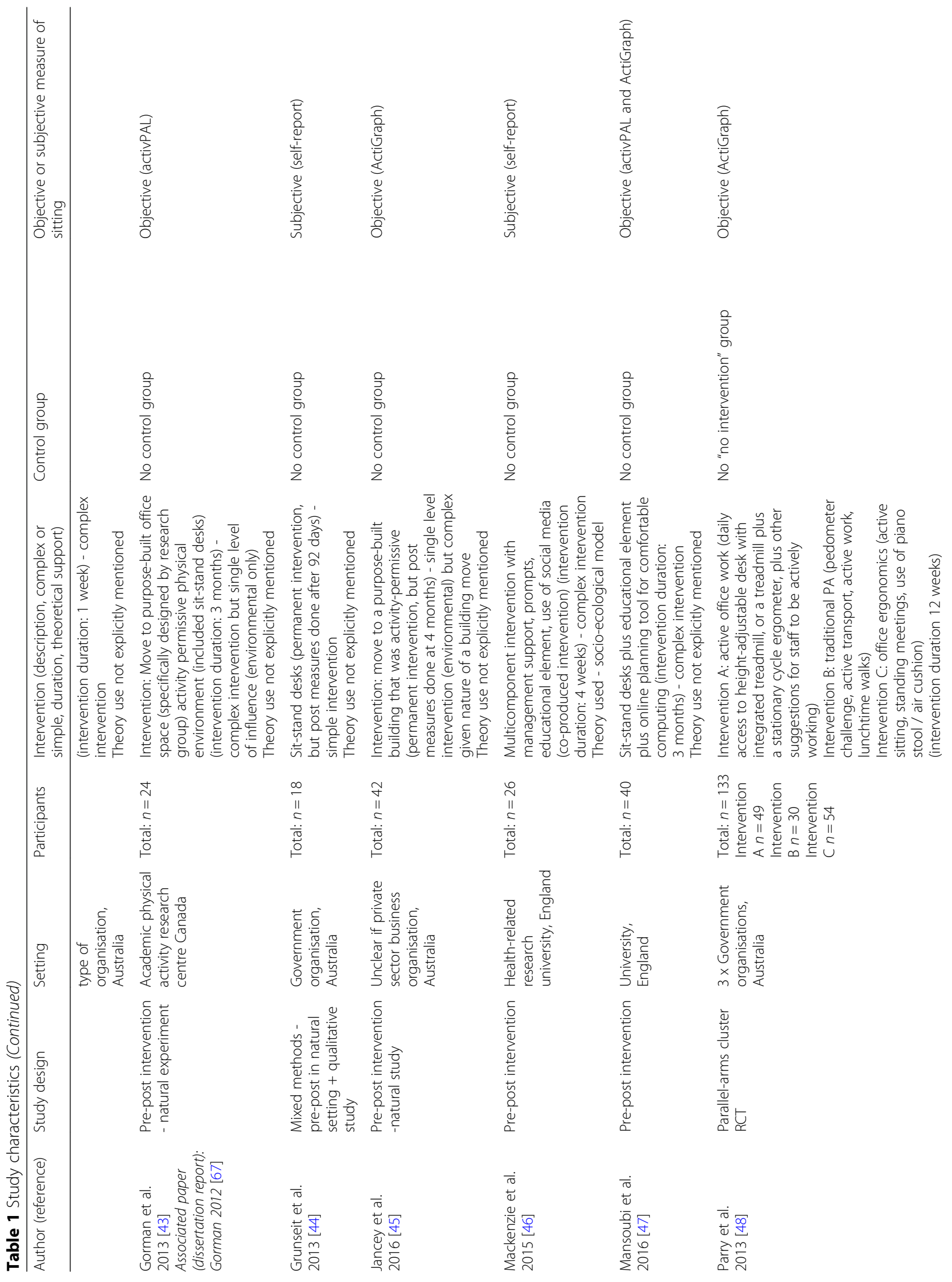




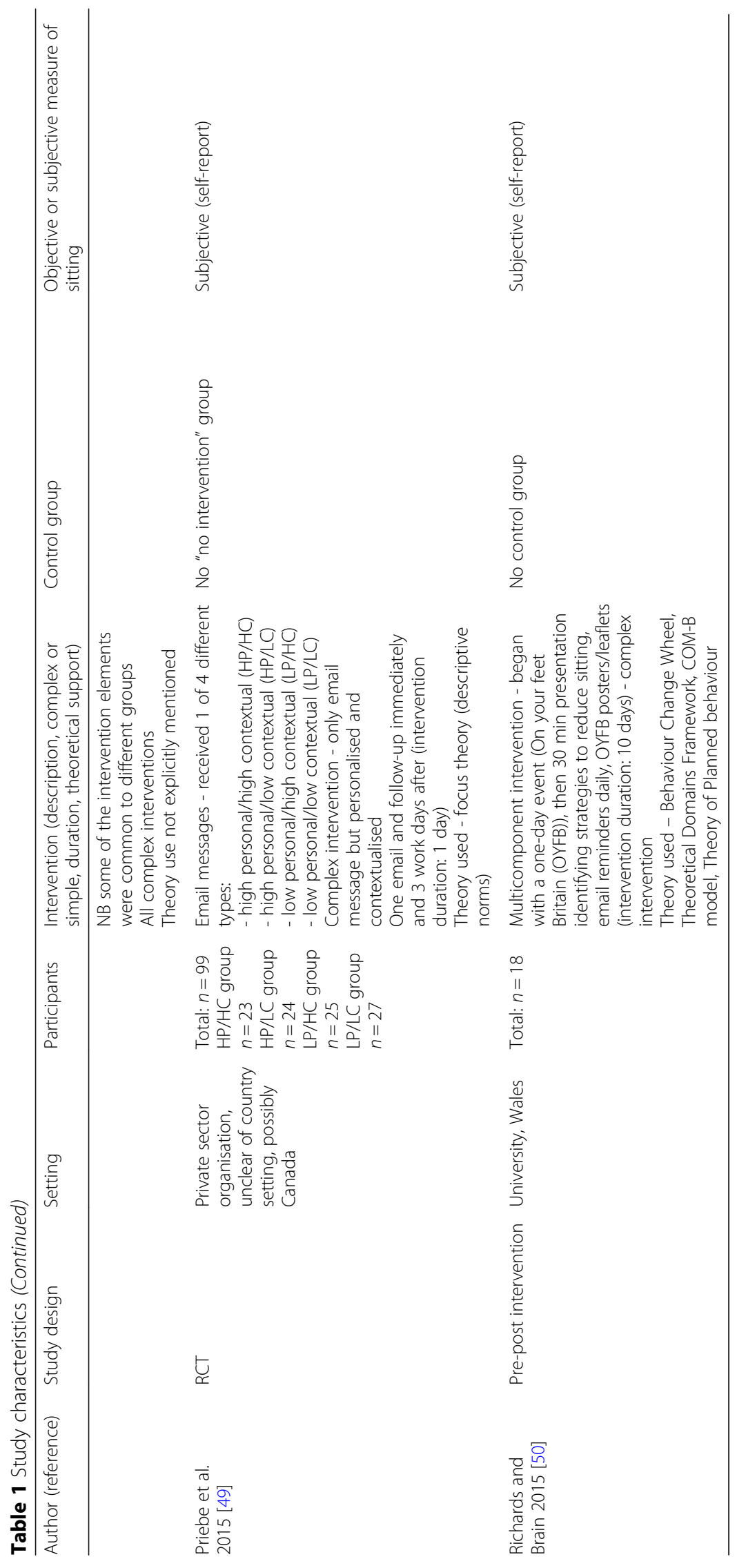


adaptation via a move to a new purpose-built building [45]; and multi-component interventions using a variety of low-cost strategies, e.g. leaflets, posters, emails, prompts, workplace champions and management support to encourage staff to reduce workplace sitting $[36,37,39,46,49,50,55,57]$.

\section{Outcome measures}

Twenty-two studies used an objective measure of sitting time via inclinometers (activPAL3) [23, 29, 31, 32, 35$37,40,41,43,47,51,53,55,57]$, and/or accelerometers (e.g., ActiGraph) [38, 42, 45, 47, 48, 52, 54, 57], or a sitting-pad device [39]. The remaining studies used subjective measures of sitting time via self-report/questionnaires [34, 44, 46, 49, 50, 56], ecological momentary assessment (e.g., using a paper diary to report whether they were sitting, standing or walking every $15 \mathrm{~min}$ ) [30], or experience-sampling methodology (e.g., responding to a text message sent three times per day) [33].

\section{Thematic synthesis}

Qualitative data relating to factors affecting intervention implementation were extracted from 34 of the 40 papers. The majority of the qualitative data extracted came from second-order constructs (author interpretations, statements, assumptions and ideas) from the following associated papers: three qualitative papers [62-64], one intervention development paper [61], one descriptive paper [66], one mixed-methods paper [44], and one dissertation report [50]. Other papers provided smaller amounts of useful qualitative data $[23,30-34,36-40,42$, $43,45-49,51-54,57,59,60,65,67]$.

The thematic synthesis coding process created 40 initial codes, from which emerged nine descriptive themes. Three higher-order analytic themes were then derived from these nine descriptive themes (see Table 2). Some descriptive themes were found to cut across several analytical domains.

\section{Understanding local barriers and facilitators to participation} Findings highlighted the importance of identifying and understanding local barriers and facilitators to intervention participation during the development phase. Individual-level barriers to interventions included: individual preference for a seated working style [44, 62, 67]; feelings of self-consciousness when standing due to the perception of being a distraction to seated colleagues $[30,50,60,62,64]$; the perception from staff and/or managers that sitting less initiatives could negatively impact on productivity $[44,50,60,62]$; and work-related factors such as the nature of work, workload and time $[30,46,50,60,62]$. The idea that sitting in the workplace represents a social norm [50,62] and the issue of the physical work environment providing limited opportunities to sit less $[37,38,44,46,50]$ were identified as further barriers. Some papers noted cost as a potential barrier to large scale roll-out, given the high upfront costs for interventions that included sit-stand desks [40, $44,51,64]$ or larger-scale environmental changes [43]. Several facilitators to "sit less" interventions were also highlighted including: perceived benefits to physical health, stress levels and productivity [44, 62]; and perceptions of peer $[30,44,50,64]$ and/or management support [23, 30, 31, 33, 36, 40, 51, 57].

\section{Identifying and using a theoretical model to operationalise intervention strategies}

Identifying and using one or more behaviour change theoretical model was found to be an important step during the development of intervention strategies. Nine

Table 2 Descriptive and analytic themes

\begin{tabular}{|c|c|c|c|}
\hline \multirow[b]{2}{*}{ Descriptive Themes } & \multicolumn{3}{|c|}{ Analytic Themes } \\
\hline & $\begin{array}{l}\text { Intervention } \\
\text { Development }\end{array}$ & $\begin{array}{l}\text { Intervention } \\
\text { Implementation }\end{array}$ & $\begin{array}{l}\text { Intervention } \\
\text { Evaluation }\end{array}$ \\
\hline Understanding local barriers and facilitators to participation & $x$ & & \\
\hline $\begin{array}{l}\text { Identifying and using a theoretical model to operationalise } \\
\text { intervention strategies }\end{array}$ & $x$ & & \\
\hline Using participatory or collaborative approaches & $x$ & $x$ & \\
\hline Conducting a pilot study within the target organisation & $x$ & $x$ & \\
\hline $\begin{array}{l}\text { Developing and implementing an action plan incorporating } \\
\text { key intervention characteristics }\end{array}$ & $x$ & $x$ & \\
\hline $\begin{array}{l}\text { Embedding the intervention within local policy strategies } \\
\text { or high-level management }\end{array}$ & & $x$ & \\
\hline Conducting a comprehensive process evaluation & & & $x$ \\
\hline $\begin{array}{l}\text { Conducting an outcome evaluation involving a range } \\
\text { of measures }\end{array}$ & & & $x$ \\
\hline Taking into account the "real-world" context & $x$ & $x$ & $x$ \\
\hline
\end{tabular}


studies reported the use of a theoretical model to inform intervention design. Single theories were used in four studies including: Social Ecological Model (SEM) [46, 58], focus theory [49], and Social Cognitive Theory (SCT) [57]. Combined theories were reported in five studies: SCT, Rogers' Diffusion on Innovations Theory and Goal-Setting Theory [52]; SCT and SEM [31, 32]; Theory of Planned Behaviour (TPB), with supporting concepts from both Self-Regulation Theory and Self-Determination Theory [53]; and the COM-B model (using the Behaviour Change Wheel and Theoretical Domains Framework) and TPB [50]. The use of theory ranged widely from: a simple mention of a theoretical model [37]; to describing the use of theory to support the development of interventions [31, 32]; to using theory to support intervention development and identify specific theoretical constructs and how these were operationalised [46, 53, 60]; and finally to using theory to support intervention development, implementation and process evaluation $[49,50,52,57]$. Grounding intervention development within one or more theoretical models was believed by some of the included studies and/or associated papers to enhance intervention effectiveness $[46,49,52,53,57,61]$.

\section{Using participatory or collaborative approaches}

It was reported that employee participation with development and implementation ensured that the intervention was acceptable and feasible for employees [46], supported engagement, tailoring and buy-in for the intervention [48], and highlighted and proposed ways to overcome anticipated barriers to intervention implementation [60]. Ten of the papers explicitly reported or recommended the use of participatory approaches during intervention development and/or implementation. Eight of these studies reported or recommended participation either via top-down involvement of team leaders/management [51], or bottom-up discussions with workplace champions [37] and/or groups of employees [23, 39, 46, $48,52,66]$ as part of workshops, focus groups, a workplace wellbeing committee, and information and consultation sessions. Two papers $[61,62]$ reported the use of collaborative approaches where both managers and employees were involved in intervention implementation.

\section{Conducting a pilot study within the target organisation}

Three of the studies in this review were pilot studies [23, $32,46]$, acting as precursors to larger trials. Two other papers described the use of pilot testing as part of the intervention development and/or implementation [52, 61]. The latter two papers reported the benefits of pilot testing to include: establishing what facilities were available in the workplace; understanding routines, interactions between employees and meeting frequencies; determining intervention efficacy, acceptability and feasibility; and allowing time for testing the implementation of the various intervention components. This information provided an opportunity to refine the intervention based on feedback from participants. Conducting a pilot within the organisation of interest therefore was anticipated to maximise the effectiveness of the intervention.

\section{Developing and implementing an action plan incorporating key intervention characteristics}

Developing and implementing an action plan [66] which incorporates a range of key intervention characteristics was an important finding. Key characteristics that were identified included:

- Tailoring an intervention to ensure that it meets the needs of individuals and/or the organisation and is relevant to different groups of employees within an organisation [32, 34, 38, 48]. Tailoring may be supported by the use of theoretical models [32, 60] and a participatory approach to intervention development [31, 39, 46, 48].

- Having a menu of strategies to provide more choice for both employees and employers [62], which can be developed using participatory approaches tailored to specific organisational contexts [39].

- Using multi-component intervention to target multiple levels of influence, i.e., at the individual-, social-, organisational- and environmental-level was characteristic [23, 31, 32, 38, 39, 46, 52] and reported to have the potential for a more comprehensive and sustainable change on workplace sitting time compared to single-level interventions [33, 39, 43, 57, 61].

- Involving workplace champions in the development and/or implementation of an intervention was found to be a beneficial strategy [23, 31, 37, 46, 61]. Workplace champions were reported to promote intervention messages and create a supportive culture within the organisation to aid change [66] or be agents of change, to advocate for the allocation of resources and influence organisational policy targeting workplace sitting [64].

- Ensuring interventions are low-cost which reflects the finding described above relating to cost being a barrier to intervention uptake [32, 40, 51, 62, 64].

- Considering interrupting versus replacing sitting time, as one study found that interrupting workplace sitting may be more feasible that replacing longer periods of sitting with standing [60].

Embedding the intervention within local policy strategies or high-level management

For effective intervention implementation and organisational change, some studies reported ensuring the 
intervention was supported by management and aligned with the target organisation's policies and/or strategies. This was achieved using one or more of the following initiatives: engaging management and gaining their commitment for the intervention [23, 46, 51, 59, 61, 62]; identifying and understanding an organisation's priorities or image by obtaining a clear description of the organisation's processes and structures that may relate to intervention implementation [51, 62]; and where possible, embedding the intervention within the organisation's processes, structures, policies and/or strategies [23, 38, $43,48,61]$.

Methods for engaging management reported by studies in this review were wide-ranging and included: managers being responsible for explicitly promoting the intervention [62]; using managers from relevant departments to facilitate the logistics of implementing an intervention, e.g., risk manager monitored planning and implementation, health and wellness manager co-ordinated all parties and arranged for the researchers to gain access to required local data [51]; presenting a business case to managers and gaining formalised commitment to the intervention [64, 66]; asking managers to distribute emails relating to the intervention [23, 39, 46]; and gaining managers' consent for their staff to participate in the intervention [23, 3032]. Consultation with managers also allowed the identification of organisational processes and structures that may be important in terms of intervention implementation [61]. These included information and potential changes in: job design, the physical work environment, workplace social norms, or workplace culture [23]. However, one study identified difficulties in changing organisational culture and suggested the need for stronger external support, such as the use of guidelines, as a way to support this change [48].

\section{Conducting a comprehensive process evaluation}

No studies explicitly mentioned the use of process evaluation, but it was undertaken to some degree by seven studies [31, 32, 37, 46, 50, 52, 62]. Process evaluation either encompassed an assessment of intervention feasibility and acceptability and/or a determination of intervention fidelity. Feasibility and acceptability was generally assessed using qualitative methods, e.g., focus groups $[32,46,50]$. Intervention fidelity used quantitative measures, e.g., surveys, aiming to establish: the "dose" of intervention delivered and whether it was implemented as planned; the "dose" that was received; and whether there were any harms or unintended consequences associated with the intervention [31, 32, 37, 52]. In addition, three studies explored the mechanisms of change by considering personal and organisational motivations which led to initial and continued participation in "sit less" interventions [44, 62, 64]. Examples of these motivations included: curiosity to try something new; interest in potential health benefits and/or experiencing changes in health outcomes; perceived improvements in productivity and energy levels; personal challenge; relevance to employees' organisation's priorities; developing task- and time-based routines, e.g., certain tasks were easier to undertake whilst not sitting and time acted as a trigger/prompt to sit less; and an awareness of the issues associated with prolonged sitting at work, which led to a shift in the perspectives of peers or managers and/or a change in organisational culture, providing employees with informal "permission" to sit less at work.

\section{Conducting an outcome evaluation involving a range of measures}

Measuring sitting/standing/moving time both within and outside of the workplace was believed to be important for intervention evaluation due to the possibility of a compensation effect, e.g., a reduction in sitting time at work resulting in an increase in sitting time at home [47, 67]. Addressing wider reaching outcomes was undertaken by some studies in this review to support a greater understanding of additional impacts of workplace sitting interventions. These included: the impact on physical (primarily musculoskeletal effects) [33, 46, 50, 62-64] and mental health and wellbeing $[46,50,64]$; work-related factors such as changes to productivity, alertness and concentration [30, 46, 50, 57, 62-64, 67]; staff morale and autonomy with feelings of empowerment to change workplace sitting behaviour [62, 64]; and wider socio-environmental changes or shifts in organisational culture $[23,30,46,50,62,63]$. Finally, including a measure of intervention cost in order to allow an assessment of return on investment, which balances costs with potential productivity trade-offs, was found to be a potential facilitator to intervention uptake and therefore an important outcome to evaluate [51]. However, none of the studies reported formal cost-effectiveness data. Four papers briefly reported the costs for a single sit-stand desk as US\$400-900 [54], $£ 360-375$ [30], US\$400 [23], US\$499 [32]. Eight papers identified that the intervention was "low-cost" [31, 34, $46,49,50]$, "a low resource intensive intervention" [37] or less expensive than more resource intensive individual-level interventions [38, 52], without presenting any quantitative cost data.

\section{Taking into account the "real-world" context}

There was a paucity of contextual information reported in the included studies. Most of the studies presented information on the type of organisation(s) participating in the study and the sector within which that organisation was based. Only a few studies reported additional contextual factors such as information of different 
occupational roles or tasks $[39,48,52]$, the organisation's prior interest/involvement in workplace health initiatives [33, 37], local organisation processes [48], and the physical work environment [52, 67]. Typically, this contextual information was only briefly described. Two papers provided some in-depth contextual information relating to the varying job roles, local organisation processes and expectations of employees [48], and the physical environments of participating workplaces [67]. However, no study explicitly included information on the hierarchical structure, the organisational culture, and the wider political and economic landscape. Therefore, it was not possible to gain a clear picture of the contexts within which the interventions were developed, implemented and evaluated.

\section{Operational intervention framework}

The findings of this review were translated into an operational framework to guide the future development, implementation and evaluation of interventions to reduce workplace sitting time (see Fig. 2). This framework displays the higher-order analytical themes within three large boxes labelled as development, implementation and evaluation. The associated lower-order descriptive themes were translated into strategies to overcome the issues or to incorporate elements of good practice as highlighted above.

\section{Discussion}

The first aim of this systematic review was to identify key considerations for the implementation of interventions to reduce workplace sitting time. Based on the qualitative evidence synthesis, three higher-order themes relating to the development, implementation and evaluation of "sit less" interventions, and nine associated lower-order descriptive themes emerged. Many of the lower-order descriptive themes align with strategies identified by the MRC for the development and evaluation of complex interventions [68]. For example, exploring barriers and facilitators to intervention development, involving stakeholders/users during intervention development and implementation, conducting a pilot study, undertaking a thorough a process and outcome evaluation and understanding contextual factors [68]. In addition, MRC guidance recommends the use of a theory-driven approach during the development of

\section{Development}

Engage with employees / managers to understand

barriers and facilitators to sitting less, e.g.:

- Nature of work, workload, time, individual working style

- Feeling self-conscious / distracting others Physical health, stress, impact on productivity

Peer and/or management support, social norms Existing work environment

Cost of an intervention

Identify and use an appropriate theoretical model to operationalise intervention strategies, e.g.:

- Socio-Ecological Model, Social Cognitive Theory, the COM-B model (Capability, Opportunity, Motivation), Rogers' Diffusion on Innovations Theory, Theory of Planned Behaviour

\section{Implementation}

Ensure intervention is supported by managemen and aligns with local policies and/or strategies:

- Management consultation - gain high-level buyin to support intervention, e.g. managers to explicitly promote intervention, facilitate logistics, send intervention emails, give consent for staff to participate

- Identify and understand organisation's priorities / image by consulting with management requires an understanding of the organisation's processes and structures

- Intervention needs to become embedded within the organisation, e.g. via changes to job design, working environment, social norms / workplace culture, or with the production of guidelines

Use participatory and/or collaborative approaches:

- Involving management - linking in with management consultation above

Involving employees - promote tailoring and buy-in, e.g. using workshops, focus groups and workplace champions; setting up a workplace wellbeing committee, running consultation events

Involving employees and management - balances a top-down and bottom-up approach

\section{Conduct a pilot study within the target organisation:}

To provide an understanding of the workplace facilities, routines and the organisational structures; to establish intervention efficacy, acceptability and feasibility; to refine intervention prior to full roll-out

Develop and implement an action plan incorporating the following key intervention characteristics:

- Tailor intervention to the needs of employees and organisation - ensuring it is applicable and relevant and can adapt to different groups of staff (linking in with the use of theory and a participatory approach)

- Provide a menu of strategies

- Target multiple levels of influence (individual, social, organisational, environmental)

Workplace champions to promote intervention messages, support organisational change, advocate and influence

Low-cost

Consider interrupting sitting vs replacing prolonged sitting with standing/moving

\section{Evaluation}

Evaluate the process:

- Assessment of feasibility and acceptability

Assessment of intervention fidelity

Implemented as planned

Received as planned

Assessment of harms / unintended consequences

Mechanisms / process of change for initial and continued participation in intervention, e.g.: Curiosity to try something new Health and work-related benefits perception of, or changes experienced Personal challenge

Task- and time-based routines, developing habits - certain tasks are easier to complet whilst not sitting; time can act as a trigger e.g. moving away from desk once per hour Relevance to organisation's priorities Raise individual / organisational awareness, leading to a change in perspective / culture

Evaluation outcomes:

Sitting, standing and moving time, measured both within and outside of the workplace to look for a compensation effect, i.e. does sitting less at work lead to more sitting outside work

Health - physical (mainly musculoskeletal reported) and mental

Work-related-productivity, concentration, energy levels

Socio-environmental / cultural changes

Employee morale and autonomy / sense of empowerment

Long-term cost-effectiveness analysis / return on investment

Take into account "real-world" contextual factors:

- Have a good understanding of the local organisation processes and structures and wider policy issues

Provide a detailed description of intervention development, implementation and evaluation processes to allow replication of methods

Fig. 2 "Sit less" intervention development, implementation and evaluation operational framework 
complex behaviour change interventions [68]. However, this review highlighted the general lack of use, or detailed reporting of, a theoretical underpinning during intervention development, with only nine of the studies reviewed addressing theoretical constructs. Further, these studies used a range of theories with no single theory appearing dominant, although it is notable that the theories largely drew from the psychological and behavioural sciences. The socially-situated nature of sedentary behaviour also provides an opportunity for the use of more sociological or organisational cultural approaches [69]. Despite the appearance of descriptive themes consistent with MRC guidance, they were only reported by a minority of studies.

The second aim was to develop an operational framework which may help to facilitate a more structured approach to developing, implementing and evaluating workplace sitting interventions and ensure consideration is given to all of the nine descriptive themes. This framework not only summarises the findings of this review, but also represents an original contribution to the knowledge base, which now requires testing in order to refine and build on this initial version. It is possible that there may be themes missing from this analysis and the operational framework. This was demonstrated by the under-reporting of contextual and process-oriented factors. Contextual factors which may be important include details of local organisation processes and structures, e.g., size of organisation, sector, hierarchical structure and organisational culture, as well as the wider political and economic landscape [69]. A recent paper looking at the social ecological correlates of objectively measured workplace sedentary behaviour found that there are work-specific individual, cultural, environmental and organisational factors associated with sedentary behaviours in the workplace and that these associations vary by job type and sector [70]. Details relating to local policy, the wider political landscape, economic issues, the physical environment, and hierarchical structures and organisational culture, would all make it possible to understand and evaluate how the intervention effects may be impacted by one, or a combination, of these factors [71, 72]. Furthermore, the readiness for change of the organisation, and the quality of existing policies and practices employed by the organisation are all key functions of effective intervention implementation [72]. It is possible that workplace contextual factors were considered during intervention development and implementation but simply not reported in some studies.

Having a detailed understanding of the context will support effective intervention implementation, a pre-requisite for effectiveness [72] and, if comprehensively reported, will aid knowledge translation into "real-world" settings. Therefore, at present, it is intended that the operational framework developed in this review be used by researchers as a prompt to ensure considerations are explicitly given to the key factors of intervention development, implementation and evaluation, which is then comprehensively reported. Once tested and refined, the framework will then be translated into as a practical tool for organisations to guide their own "sit less" intervention development, implementation and evaluation.

Most of the studies included in the review were RCTs and used objective measurement tools (e.g., activPAL inclinometers). This represents an improvement in study design compared to previous reviews $[17,20]$. However, this review demonstrates the importance of gathering context-rich and process-oriented data to more fully understand the factors that may promote or impede intervention effectiveness. The participants of the included studies were mainly well-educated females. This may be due to the most common setting for these studies being within academic institutions which employ large numbers of tertiary educated staff and because physical activity interventions tend to attract mainly female participants [73]. Therefore, future research should seek to develop and evaluate workplace sitting interventions outside of academic institutions.

The current review has a number of strengths. First, this review is the first to undertake a qualitative assessment of interventions to reduce workplace sitting. Second, due to the need to obtain rich qualitative data, a wide range of studies were included, allowing a broad understanding of factors relating to the development, implementation and evaluation of interventions aimed at reducing workplace sitting time. Third, the qualitative evidence synthesis provided the basis for the development of an evidence-based operational framework, which after a period of testing and refinement, could be translated into a practical toolkit for use by a range of organisations, thereby supporting knowledge mobilisation into "real-world" settings.

The review findings should also be considered in the context of several limitations. First, the search was limited to only four databases and, as a result, some studies may have been missed. Second, articles were limited to those published in English, which may have resulted in relevant articles published in other languages being missed. However, to ensure all relevant papers were identified, the lead author conducted a citation search of all systematic reviews that synthesised intervention studies with similar inclusion/exclusion criteria. The citation search did not identify any non-English intervention studies. Third, the qualitative evidence synthesis and subsequent framework development is limited to the qualitative data within the published papers. There were papers where no qualitative data were extracted due to a lack of reporting of factors relating to the 
implementation of interventions. It is likely that there are insights from these studies that could also be used to further inform the framework. Future research should aim to provide more detailed reporting of factors relating to intervention development, implementation and evaluation.

\section{Conclusions}

This qualitative systematic review explored factors that aid the effective implementation of workplace sitting interventions. The findings indicate a need for comprehensive intervention development, implementation and evaluation processes which should focus on a range of strategies including: understanding the barriers and facilitators to participating in workplace sitting interventions; identification and use of a theoretical model; gaining management support and ensuring an intervention aligns with existing policies/strategies; the use of participatory approaches; conducting a pilot study; developing and implementing an action plan; and undertaking a comprehensive process and outcome evaluation. In addition, an under-reported cross-cutting consideration relates to the consideration of the context within which interventions are undertaken. The qualitative synthesis findings led to an operational framework to inform the planning of future "sit less" interventions. This framework needs to be formally tested in a range of workplace settings to establish whether it is fit for purpose and whether it adequately captures all relevant contextual factors. This work, in turn, will help to ensure that the potential of the framework to inform the development, implementation and evaluation of effective interventions to reduce workplace sitting time is realised.

\section{Additional files}

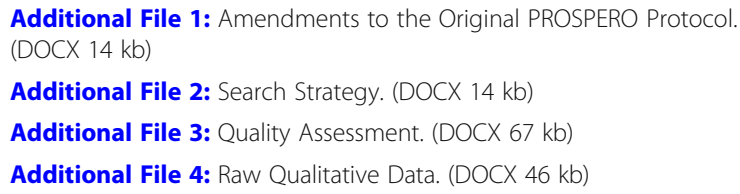

\section{Abbreviations}

COM-B : Capability Opportunity Motivation Behaviour Change Model; MRC: Medical Research Council; PRISMA : Preferred Reporting Items for Systematic reviews and Meta-analyses; RCT : Randomised-Controlled Trials; SCT : Social Cognitive Theory; SEM : Social Ecological Model; TPB : Theory of Planned Behaviour

\section{Funding}

This report is independent research arising from a Doctoral Research Fellowship of the lead author, Kelly Mackenzie DRF-2016-09-023, supported by the National Institute for Health Research. The views expressed in this publication are those of the authors and not necessarily those of the NHS, the National Institute for Health Research, Health Education England or the Department of Health. The funding body was not involved in the design of the study, or the collection, analysis, and interpretation of data, or in writing the manuscript.
Availability of data and materials

All data generated or analysed during this study are included in this published article [and its supplementary information files].

\section{Authors' contributions}

KM originally conceived the review idea. All authors (KM, ES, PN and EG) contributed to developing the research question, devising the methods, formalising the results and developing the operational framework. KM conducted the search strategy with support from ES and PN, and KM undertook the data analysis with support from ES. KM was the major contributor in writing the manuscript, but EG, PN and ES read and commented upon drafts and approved the final manuscript.

\section{Authors' information}

KM is funded by a Doctoral Research Fellowship (DRF) from the National Institute for Health Research. This systematic review is part of the first phase of the DRF. She now intends to use the findings from this review in future phases of her DRF which will include the development, implementation and evaluation of interventions to reduce workplace sitting in four different organisations

ES, PN and EG are all supervising this DRF.

Ethics approval and consent to participate

Not applicable.

Consent for publication

Not applicable.

\section{Competing interests}

The authors declare that they have no competing interests.

\section{Publisher's Note}

Springer Nature remains neutral with regard to jurisdictional claims in published maps and institutional affiliations.

\section{Author details}

${ }^{1}$ School of Health and Related Research, University of Sheffield, Regent Street, Sheffield S1 4DA, UK. ${ }^{2}$ Department of Psychology, University of Sheffield, Cathedral Court, 1 Vicar Lane, Sheffield S1 2LT, UK

Received: 23 March 2018 Accepted: 27 June 2018

Published online: 04 July 2018

References

1. Tremblay MS, Aubert S, Barnes JD, Saunders TJ, Carson V, Latimer-Cheung $A E$, et al. Sedentary behavior research network (SBRN) - terminology consensus project process and outcome. Int J Behav Nutr Phys Act. 2017;14: 75. Available from: https://ijbnpa.biomedcentral.com/track/pdf/10.1186/ s12966-017-0525-8?site=ijbnpa.biomedcentral.com. [cited 2017 Sep 12].

2. Owen N, Healy GN, Matthews CE, Dunstan DW. Too much sitting: the population health science of sedentary behavior. Exerc Sport Sci Rev. 2010;38: 105-13. Available from: http://www.pubmedcentral.nih.gov/articlerender. fcgi?artid=3404815\&tool=pmcentrez\&rendertype=abstract.[cited 2015 Jul 21]

3. Dunstan DW, Howard B, Healy GN, Owen N. Too much sitting: a health hazard. Diabetes Res Clin Pract. 2012;97:368-76. Available from: http://www. ncbi.nlm.nih.gov/pubmed/22682948. [cited 2015 May 19].

4. Ford ES, Caspersen CJ. Sedentary behaviour and cardiovascular disease: a review of prospective studies. Int J Epidemiol. 2012;41:1338-53. Available from: http://ije.oxfordjournals.org/content/41/5/1338.short. [cited 2015 Jul 1].

5. Grøntved A, Hu FB. Television viewing and risk of type 2 diabetes, cardiovascular disease, and all-cause mortality: a meta-analysis. JAMA. 2011; 305:2448-55. Available from: http://jama.jamanetwork.com/article. aspx?articleid=900893. [cited 2015 Nov 17].

6. Alavi S, Makarem J, Mehrdad R, Abbasi M. Metabolic syndrome: a common problem among office workers. Int J Occup Env Med. 2015;6:492-534. Available from: http://www.theijoem.com/ijoem/index.php/ijoem/article/ view/492/588. [cited 2015 May 15].

7. Edwardson CL, Gorely T, Davies MJ, Gray LJ, Khunti K, Wilmot EG, et al. Association of sedentary behaviour with metabolic syndrome: a meta-analysis. PLoS One. 2012;7:e34916. Available from: https://doi.org/10.1371/journal.pone. 0034916. [cited 2015 Jul 1]. 
8. Proper Kl, Singh AS, van Mechelen W, Chinapaw MJM. Sedentary behaviors and health outcomes among adults: a systematic review of prospective studies. Am J Prev Med. 2011;40:174-82. Available from: http://www. sciencedirect.com/science/article/pii/S0749379710006082. [cited 2015 Jul 10].

9. Brown WJ, Miller YD, Miller R. Sitting time and work patterns as indicators of overweight and obesity in Australian adults. Int J Obes Relat Metab Disord. 2003;27:1340-1346. Available from: https://doi.org/10.1038/sj.ijo.0802426. [cited 2015 Jul 1].

10. Lee PH, FKY W. The association between time spent in sedentary behaviors and blood pressure: a systematic review and meta-analysis. Sports Med. 2015:45:867-80. Available from: http://link.springer.com/10.1007/s40279-0150322-y. [cited 2015 Nov 17].

11. Lynch BM. Sedentary behavior and cancer: a systematic review of the literature and proposed biological mechanisms. Cancer Epidemiol Biomarkers Prev. 2010;19:2691-709. Available from: http://cebp.aacrjournals.org/content/early/ 2010/10/22/1055-9965.EPI-10-0815.abstract. [cited 2015 Nov 17].

12. Teychenne M, Ball K, Salmon J. Sedentary behavior and depression among adults: a review. Int J Behav Med. 2010;17:246-54. Available from: http:// www.ncbi.n/m.nih.gov/pubmed/20174982. [cited 2015 Jun 22].

13. Mörl F, Bradl I. Lumbar posture and muscular activity while sitting during office work. J Electromyogr Kinesiol. 2013;23:362-8. Available from: http:// www.ncbi.n/m.nih.gov/pubmed/23122693. [cited 2015 Jan 30].

14. Katzmarzyk PT, Church TS, Craig CL, Bouchard C. Sitting time and mortality from all causes, cardiovascular disease, and cancer. Med Sci Sports Exerc 2009;41:998-1005. Available from: http://citeseerx.ist.psu.edu/viewdoc/ download?. [cited 2015 Aug 20].

15. Church TS, Thomas DM, Tudor-Locke C, Katzmarzyk PT, Earnest CP, Rodarte $R Q$, et al. Trends over 5 decades in U.S. occupation-related physical activity and their associations with obesity. PLoS One. 2011;6:e19657. Available from: http://dx.plos.org/10.1371/journal.pone.0019657. [cited 2017 Sep 12].

16. Clemes SA, Patel R, Mahon C, Griffiths PL. A T, A B. Sitting time and step counts in office workers. Occup Med (Chic III). 2014;64:188-92. Available from: https://academic.oup.com/occmed/article-lookup/doi/10.1093/ occmed/kqt164. [cited 2017 Jul 3].

17. Chu AHY, Ng SHX, Tan CS, Win AM, Koh D, Müller-Riemenschneider F. A systematic review and meta-analysis of workplace intervention strategies to reduce sedentary time in white-collar workers. Obes Rev. 2016;17:46781. Available from: http://www.ncbi.n/m.nih.gov/pubmed/26990220. [cited 2016 Apr 7]

18. Commissaris DA, Huysmans MA, Mathiassen SE, Srinivasan D, Koppes LL, Hendriksen IJ. Interventions to reduce sedentary behavior and increase physical activity during productive work: a systematic review. Scand J Work Environ Health. 2016;42:181-91. Available from: https://www.ncbi.nlm.nih. gov/pubmed/26683116.

19. Hutcheson AK, Piazza AJ, Knowlden AP. Work site-based environmental interventions to reduce sedentary behavior: a systematic review. Am J Health Promot. 2016;32(1):32-47. Available from: http://journals.sagepub. com/doi/abs/10.1177/0890117116674681.

20. Shrestha N, ljaz S, Kukkonen-Harjula KT, Kumar S, Nwankwo CP. Workplace interventions for reducing sitting at work. Cochrane database Syst Rev. 2015;1:CD010912. Available from: http://www.ncbi.n/m.nih.gov/pubmed/ 25620219. [cited 2015 Jul 2].

21. Tew GA, Posso MC, Arundel CE, McDaid CM. Systematic review: heightadjustable workstations to reduce sedentary behaviour in office-based workers. Occup Med (Lond) 2015;kqv044-. Available from: http://occmed. oxfordjournals.org/content/early/2015/04/29/occmed.kqv044.short. [cited 2015 Jun 10].

22. Neuhaus M, Eakin EG, Straker L, Owen N, Dunstan DW, Reid N, et al. Reducing occupational sedentary time: a systematic review and metaanalysis of evidence on activity-permissive workstations. Obes Rev. 2014;15: 822-38. Available from: http://www.ncbi.nlm.nih.gov/pubmed/25040784 [cited 2015 May 6].

23. Healy GN, Eakin EG, Lamontagne AD, Owen N, Winkler EAH, Wiesner G, et al. Reducing sitting time in office workers: short-term efficacy of a multicomponent intervention. Prev Med (Baltim). 2013;57:43-8. Available from: http://www.ncbi.nlm.nih.gov/pubmed/23597658. [cited 2015 Apr 30]

24. Thorp AA, Kingwell BA, Owen N, Dunstan DW. Breaking up workplace sitting time with intermittent standing bouts improves fatigue and musculoskeletal discomfort in overweight/obese office workers. Occup Environ Med. 2014;71:765-71. Available from: http://oem.bmj.com/content/ 71/11/765.short. [cited 2015 Aug 12].
25. Moore GF, Audrey S, Barker M, Bond L, Bonell C, Hardeman W, et al. Process evaluation of complex interventions: Medical Research Council guidance. BMJ. 2015;350:h1258. Available from: http://www.bmj.com/content/350/bmj. h1258. [cited 2017 Jun 9].

26. Moher D, Liberati A, Tetzlaff J, Altman DG, Altman D. Preferred reporting items for systematic reviews and meta-analyses: the PRISMA statement. PLoS Med. 2009;6:e1000097. Available from: http://dx.plos.org/10.1371/ journal.pmed.1000097. [cited 2017 Jun 28].

27. Thomas J, Harden A. Methods for the thematic synthesis of qualitative research in systematic reviews. BMC Med Res Methodol. 2008;8:45. Available from: http://www.biomedcentral.com/1471-2288/8/45. [cited 2017 Jun 26].

28. Dixon-Woods M, Bonas S, Booth A, Jones DR, Miller T, Sutton AJ, et al. How can systematic reviews incorporate Qual Res? A critical perspective. Qual Res. 2006;6:27-44. Available from: http://qrj.sagepub.com/cgi/doi/10.1177/ 1468794106058867. [cited 2017 Jun 26].

29. Alkhajah TA, Reeves MM, Eakin EG, Winkler EAH, Owen N, Healy GN. Sitstand workstations: a pilot intervention to reduce office sitting time. Am J Prev Med. 2012;43:298-303. Available from: http://www.ncbi.nlm.nih.gov/ pubmed/22898123. [cited 2015 Apr 30].

30. Graves LE, Murphy RC, Shepherd SO, Cabot J, Hopkins ND. Evaluation of sitstand workstations in an office setting: a randomised controlled trial. BMC Public Health. 2015;15:1145. Available from: http://bmcpublichealth. biomedcentral.com/articles/10.1186/s12889-015-2469-8. [cited 2015 Nov 23].

31. Healy GN, Eakin EG, Owen N, Lamontagne AD, Moodie M, Winkler EAH, et al. A cluster randomized controlled trial to reduce office workers' sitting time: effect on activity outcomes. Med Sci Sports Exerc. 2016:48:1787-97. Available from: https://www.ncbi.nlm.nih.gov/pubmed/27526175.

32. Neuhaus M, Healy G, Dunstan D. Workplace sitting and height-adjustable workstations: a randomized controlled trial. Am J Prev Med. 2014. Available from: http://www.sciencedirect.com/science/article/pii/S0749379713005369. [cited 2015 Mar 19].

33. Pronk NP, Katz AS, Lowry M, Payfer JR. Reducing occupational sitting time and improving worker health: the Take-a-Stand Project, 2011. Prev Chronic Dis. 2012;9:-E154. Available from: http://www.pubmedcentral.nih.gov/ articlerender.fcgi?artid=3477898\&tool=pmcentrez\&rendertype=abstract. [cited 2015 Jul 1].

34. Puig-Ribera A, Bort-Roig J, Gonzalez-Suarez AM, Martinez-Lemos I, GineGarriga M, Fortuno J, et al. Patterns of impact resulting from a 'sit less, move more' web-based program in sedentary office employees. PLoS One 2015;10:e0122474. https://doi.org/10.1371/journal.pone.0122474.

35. Tobin R, Leavy J, Jancey J. Uprising: an examination of sit-stand workstations, mental health and work ability in sedentary office workers, in Western Australia. Work. 2016:55:359-71. Available from: https://www.ncbi. nlm.nih.gov/pubmed/27689593.

36. Urda JL, Lynn JS, Gorman A, Larouere B. Effects of a minimal workplace intervention to reduce sedentary behaviors and improve perceived wellness in middle-aged women office workers. J Phys Act Health. 2016;13:838-44. Available from: https://www.ncbi.nlm.nih.gov/pubmed/26998705.

37. Brakenridge CL, Fjeldsoe BS, Young DC, Winkler EAH, Dunstan DW, Straker LM, et al. Evaluating the effectiveness of organisational-level strategies with or without an activity tracker to reduce office workers' sitting time: a cluster-randomised trial. Int J Behav Nutr Phys Act. 2016;13:115. Available from: https://ijbnpa.biomedcentral.com/articles/10.1186/s12966-016-0441-3.

38. Donath L, Faude O, Schefer Y, Roth R, Zahner L. Repetitive daily point of choice prompts and occupational sit-stand transfers, concentration and neuromuscular performance in office workers: an RCT. Int J Environ Res Public Health. 2015;12:4340-53

39. Gilson ND, Ng N, Pavey TG, Ryde GC, Straker L, Brown WJ. Project Energise: Using participatory approaches and real time computer prompts to reduce occupational sitting and increase work time physical activity in office workers. J Sci Med Sport. 2016;19:926-30. Available from: https://www.ncbi. nlm.nih.gov/pubmed/26922132.

40. Chau JY, Daley M, Dunn S, Srinivasan A, Do A, Bauman AE, et al. The effectiveness of sit-stand workstations for changing office workers' sitting time: results from the stand@work randomized controlled trial pilot. Int J Behav Nutr Phys Act. 2014;11:127. Available from: http://www. pubmedcentral.nih.gov/articlerender.fcgi?artid=4194364\&tool= pmcentrez\&rendertype=abstract. [cited 2015 Jan 23].

41. Swartz AM, Rote AE, Welch WA, Maeda H, Hart TL, Cho Yl, et al. Prompts to disrupt sitting time and increase physical activity at work, 2011-2012. Prev Chronic Dis. 2014;11:E73. Available from: http://www.pubmedcentral.nih. 
gov/articlerender.fcgi?artid=4008949\&tool=pmcentrez\&rendertype=abstract. [cited 2015 May 19].

42. Gilson ND, Suppini A, Ryde GC, Brown HE, Brown WJ. Does the use of standing "hot" desks change sedentary work time in an open plan office? Prev Med (Baltim). 2012;54:65-7. Available from: http://www.sciencedirect. com/science/article/pii/S0091743511004300. [cited 2015 Jul 1].

43. Gorman E, Ashe MC, Dunstan DW, Hanson HM, Madden K, Winkler EAH, et al. Does an "activity-permissive" workplace change office workers' sitting and activity time? PLoS One. 2013;8:e76723. Available from: http://www. pubmedcentral.nih.gov/articlerender.fcgi?artid=3788722\&tool= pmcentrez\&rendertype=abstract. [cited 2015 Feb 25].

44. Grunseit AC, Chau JY-Y, van der Ploeg HP, Bauman A. "Thinking on your feet": a qualitative evaluation of sit-stand desks in an Australian workplace. BMC Public Health. 2013;13:365

45. Jancey JM, McGann S, Creagh R, Blackford KD, Howat P, Tye M. Workplace building design and office-based workers' activity: a study of a natural experiment. Aust N Z J Public Health. 2016;40:78-82. Available from: http:// www.ncbi.nlm.nih.gov/pubmed/26455349 [cited 2016 Feb 22].

46. Mackenzie K, Goyder E, Eves F. Acceptability and feasibility of a low-cost, theory-based and co-produced intervention to reduce workplace sitting time in desk-based university employees. BMC Public Health. 2015;15:1294. Available from: https://www.ncbi.nlm.nih.gov/pmc/articles/PMC4690339/.

47. Mansoubi M, Pearson N, Biddle SJH, Clemes SA. Using sit-to-stand workstations in offices: is there a compensation effect? Med Sci Sports Exerc. 2016;48:720-5. Available from: http://europepmc.org/abstract/med/ 26496419 [cited 2016 May 3]

48. Parry S, Straker L, Gilson ND, Smith AJ. Participatory workplace interventions can reduce sedentary time for office workers: a randomised controlled trial. PLoS One. 2013;8:e78957. Available from: http://www.pubmedcentral.nih. gov/articlerender.fcgi?artid=3827087\&tool=pmcentrez\&rendertype=abstract. [cited 2015 Feb 10].

49. Priebe CS, Spink KS. Less sitting and more moving in the office: Using descriptive norm messages to decrease sedentary behavior and increase light physical activity at work. Psychol Sport Exerc. 2015;19:76-84. Available from: http://www.sciencedirect.com/science/article/pii/S1469029215000230. [cited 2015 Apr 3]

50. Richards R, Brain K. Small-scale feasibility study to evaluate the effects of the 'On Your Feet Britain' campaign in a High Educ institution setting. Cardiff: University of Cardiff; 2015.

51. Chau JY, Sukala W, Fedel K, Do A, Engelen L, Kingham M, et al. More standing and just as productive: effects of a sit-stand desk intervention on call center workers' sitting, standing, and productivity at work in the Opt to Stand pilot study. Prev Med reports. 2016;3:68-74. Available from: https://www.ncbi.nlm. nih.gov/pubmed/26844191.

52. Danquah IH, Kloster S, Holtermann A, Aadahl M, Bauman A, Ersboll AK, et al. Take a Stand!-a multi-component intervention aimed at reducing sitting time among office workers - a cluster randomized trial. Int J Epidemiol. 2016;46(1):30-40. Available from: https://www.ncbi.nlm.nih.gov/pubmed/ 27094749.

53. De Cocker K, De Bourdeaudhuij I, Cardon G, Vandelanotte C. The effectiveness of a web-based computer-tailored intervention on workplace sitting: a randomized controlled trial. J Med Internet Res. 2016;18:e96. Available from: https://www.ncbi.nlm.nih.gov/pubmed/27245789.

54. Dutta N, Koepp G, Stovitz S, Levine J, Pereira M. Using sit-stand workstations to decrease sedentary time in office workers: a randomized crossover trial. Int J Environ Res Public Health. 2014;11:6653-65. Available from: http:// www.pubmedcentral.nih.gov/articlerender.fcgi?artid=4113835\&tool= pmcentrez\&rendertype=abstract. [cited $2015 \mathrm{Mar}$ 17].

55. Evans RE, Fawole HO, Sheriff SA, Dall PM, Grant PM, Ryan CG. Point-ofchoice prompts to reduce sitting time at work: a randomized trial. Am J Prev Med. 2012;43:293-7. Available from: http://www.ncbi.nIm.nih.gov/ pubmed/22898122. [cited 2015 May 19].

56. Gao Y, Nevala N, Cronin NJ, Finni T. Effects of environmental intervention on sedentary time, musculoskeletal comfort and work ability in office workers. Eur J Sport Sci. 2016;16:747-54. Available from: https://www.ncbi. nlm.nih.gov/pubmed/26529590.

57. Gordon A. A theory-based pilot study to decrease sitting time in the workplace - Dissertation Thesis. Arizona: Arizona State University; 2013.

58. Brakenridge CL, Fjeldsoe BS, Young DC, Winkler EAH, Dunstan DW, Straker LM, et al. Organizational-level strategies with or without an activity tracker to reduce office workers' sitting time: rationale and study design of a pilot cluster-randomized trial. JMIR Res Protoc. 2016;5:e73. Available from: https:// www.ncbi.nlm.nih.gov/pubmed/27226457.

59. Dunstan DW, Wiesner G, Eakin EG, Neuhaus M, Owen N, LaMontagne AD, et al. Reducing office workers' sitting time: rationale and study design for the stand up Victoria cluster randomized trial. BMC Public Health. 2013;13:1057. Available from: http://www.pubmedcentral.nih.gov/articlerender.fcgi?artid= 3828481\&tool=pmcentrez\&rendertype=abstract. [cited 2015 Mar 5].

60. De Cocker K, De Bourdeaudhuij I, Cardon G, Vandelanotte C. Theory-driven, web-based, computer-tailored advice to reduce and interrupt sitting at work: development, feasibility and acceptability testing among employees. BMC Public Health. 2015;15:959. Available from: http://www.biomedcentral. com/1471-2458/15/959. [cited 2015 Oct 8].

61. Neuhaus M, Healy GN, Fjeldsoe BS, Lawler S, Owen N, Dunstan DW, et al. Iterative development of Stand Up Australia: a multi-component intervention to reduce workplace sitting. Int J Behav Nutr Phys Act. 2014;11:21. Available from: http://www.pubmedcentral.nih.gov/articlerender.fcgi?artid= 3936706\&tool=pmcentrez\&rendertype=abstract. [cited 2015 May 19].

62. Chau JY, Daley M, Srinivasan A, Dunn S, Bauman AE, van der Ploeg HP. Desk-based workers' perspectives on using sit-stand workstations: a qualitative analysis of the stand@work study. BMC Public Health 2014;14: 752. https://doi.org/10.1186/1471-2458-14-752.

63. Dutta N, Walton T, Pereira MA. Experience of switching from a traditional sitting workstation to a sit-stand workstation in sedentary office workers. Work. 2015;52:83-9. Available from: http://content.iospress.com/articles/ work/wor 1971. [cited 2015 Oct 23].

64. Leavy J, Jancey J. Stand by me: qualitative insights into the ease of use of adjustable workstations. AIMS PUBLIC Heal. 2016;3:644-62.

65. Stephens SK, Winkler EAH, Trost SG, Dunstan DW, Eakin EG, Chastin SFM, et al. Intervening to reduce workplace sitting time: how and when do changes to sitting time occur? Br J Sports Med. 2014;48:1037-42. Available from: http://bjsm.bmj.com/content/early/2014/05/09/bjsports-2014-093524.short. [cited 2015 May 15].

66. Healy GN, Goode A, Schultz D, Lee D, Leahy B, Dunstan DW, et al. The BeUpstanding program (TM): scaling up the stand up Australia workplace intervention for translation into practice. AIMS PUBLIC Heal. 2016;3:341-7. https://bjsm.bmj.com/content/48/13/1037.

67. Gorman E. Sedentary time in the workplace: a natural experiment of the transition to an activity-permissive workplace physical environment dissertation thesis. MSc Diss. Thesis. Vancouver: University of British Columbia; 2012

68. Craig P. Developing and evaluating complex interventions: New guidance. London: Medical Research Council; 2006.

69. Such E, Mutrie N. Using Organisational Cultural Theory to understand workplace interventions to reduce sedentary time. Int J Heal Promot Educ. 2017;55:18-29. Available from: http://eprints.whiterose.ac.uk/101513/. [cited 2018 Mar 9].

70. Mullane SL, Toledo MJL, Rydell SA, Feltes LH, Vuong B, Crespo NC, et al. Social ecological correlates of workplace sedentary behavior. Int J Behav Nutr Phys Act. 2017;14:117. Available from: https://www.ncbi.nlm.nih.gov/ pmc/articles/PMC5580289/pdf/12966_2017_Article_576.pdf. [cited 2017 Oct 19].

71. Petticrew M, Rehfuess E, Noyes J, Higgins JPT, Mayhew A, Pantoja T, et al. Synthesizing evidence on complex interventions: how meta-analytical, qualitative, and mixed-method approaches can contribute. J Clin Epidemiol. 2013;66:1230-43. Available from: http://www.ncbi.nlm.nih.gov/pubmed/ 23953082. [cited 2017 Oct 4].

72. Weiner BJ, Lewis MA, Linnan LA. Using organization theory to understand the determinants of effective implementation of worksite healt $\mathrm{h}$ promotion programs. Health Educ Res. 2008;24:292-305. Available from: https://academic. oup.com/her/article-lookup/doi/10.1093/her/cyn019. [cited 2017 Jul 2]

73. Waters LA, Galichet B, Owen N, Eakin E. Who participates in physical activity intervention trials? J Phys Act Health. 2011:8:85-103. Available from: http:// www.ncbi.nlm.nih.gov/pubmed/21297189. [cited 2017 Oct 19]. 